R. Brooks and R. Tse

Nagoya Math. J.

Vol. 117 (1990), 227

\title{
CORRECTION TO \\ "ISOSPECTRAL SURFACES OF SMALL GENUS," (NAGOYA MATH. J. VOL. 107 (1987), 13-24)
}

\section{ROBERT BROOKS AND RICHARD TSE}

It was brought to our attention by Zoran Luicic and Milica Stojanovic, via Peter Gilkey, that some of the diagrams in our paper are not correct.

The particular problems are the gluing diagrams for the pair of isospectral surfaces of genus 4 , which occur on page 20 . It is easy to check that the gluing diagrams given there give rise to a surface of the wrong genus. The problem arose because of carelessness in some of the identifications of some of the edges of the fundamental domain.

Fortunately, the problem is easily fixed. To obtain a correct gluing diagram, in each fundamental domain in each of the two figures, switch the labeling on the first and second edges, and on the third and fourth edges. Thus, the labeling on the first diagram should read, staring with the lefthand edge on the top fundamental domain and moving clockwise:

B, 1, A, 1, F, 2, C, 4, D, 5, E, 7, E, 4, F, 3, A, 3, B, 2, C, 6, D, 5, G, 7, G, 6

and on the second diagram:

A, 1, D, 1, C, 4, B, 2, E, 3, F, 4, G, 7, G, 5, D, 5, C, 6, F, 2, E, 3, B, 6, A, 7.

We would like to thank Luicic and Stojanovic for their careful reading of the paper.

Department of Mathematics

University of Southern California

Los Angeles, California 90089-1113

U.S.A.

Received October 16, 1989. 